\title{
Checking unimodality using isotonic regression: an application to breast cancer mortality rates
}

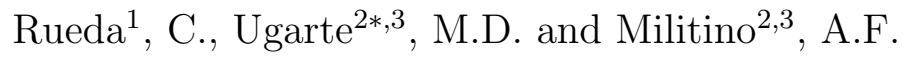 \\ ${ }^{1}$ Department of Statistics and Operations Research, Valladolid University \\ Facultad de Ciencias, 47005 Valladolid, Spain \\ ${ }^{2}$ Department of Statistics and Operations Research, Public University of Navarre \\ ${ }^{3}$ INAMAT, Public University of Navarre \\ Campus de Arrosadia, 31006 Pamplona, Spain \\ e-mail ${ }^{2 *}$ : lola@unavarra.es
}

\begin{abstract}
In some diseases it is well-known that a unimodal mortality pattern exists. A clear example in developed countries is breast cancer, where mortality increased sharply until the nineties and then decreased. This clear unimodal pattern is not necessarily applicable to all regions within a country. In this paper, we develop statistical tools to check if the unimodality pattern persists within regions using order restricted inference. Break points as well as confidence intervals are also provided. In addition, a new test for checking monotonicity against unimodality is derived allowing to discriminate between a simple increasing pattern and an up-then-down response pattern. A comparison with the widely used joinpoint regression technique under unimodality is provided. We show that the joinpoint technique could fail when the underlying function is not piecewise linear. Results will be illustrated using age-specific breast cancer mortality data from Spain in the period 1975-2005.
\end{abstract}

Keywords: change point, isotonic regression, order restricted inference, temporal trends, joint point regression, segmented regression

\section{Introduction}

The analysis of mortality and incidence trends is very informative when analyzing cancer incidence or mortality (Ruiz-Medina et al., 2014). Changes in the (temporal) trend could be due to many reasons like an improvement in the treatment of the disease, better habits or life style in the population or just the effect of a screening program. The detection of these changes in trends is then of great interest for epidemiologists and public health researchers. In the epidemiological literature, the classical procedure used to detect trend changes is called joinpoint regression. The joinpoint regression software developed by the National Cancer Institute of the United States is widely used (Molodecky et al., 2012, Simard et al., 2012, de Souza et al., 2013, Hur et al., 2013, López-Campos et al., 2013). The implemented method is developed by Kim et al. (2000), although the basics of the method are older (Sprent, 1961, Hudson, 1966, Feder, 1975, Lerman, 1980). The objective of the joinpoint technique is to find not only trend changes 
but also its location. In many applications of regression in different fields, the response and the explanatory variable exhibit a unimodal pattern, i.e., the response variable increases up to a certain unknown point and then decreases. This up-then-down response pattern is usually called an umbrella or unimodal ordering. Unimodal regression often arises in the analysis of epidemiological data, such as dose response studies, where the response increases with the dose until a certain level and then decreases. Additional examples are given by Morton-Jones et al.(2000), Banerjee et al. (2009) or Gunn and Dunson (2005). Many of these applications are aimed to locate the peak and to test the null hypothesis of a simpler increasing pattern. An interesting case is the analysis of surveillance data from an epidemic (Bock et al., 2008), where the interest relies on an early detection of the peak of the epidemic that can be accomplished with the $p$-value of the mentioned test. Different parametric approaches can be used to estimate a unimodal relationship and the corresponding peak, such as fitting a quadratic or a joinpoint regression. Nonparametric approaches can also be used. For example, Ugarte et al (2010) choose the peak as a simple choice of selecting the maximum of a smoothed version of the data and more recently Köllmann et al. (2012) use splines for the same goal. Both, the parametric and the nonparametric solutions have important drawbacks. The parametric approaches require a careful choice of the form of the function giving biased estimates of the location of the peak under an erroneous specification of the function. The nonparametric approaches are not easy to implement and require user-specified choices, such as bandwidth, smoothing parameters or the number and placement of knots. The adopted proposal of this paper is a semiparametric regression approach with specific advantages. It is very simple and it gives a location estimate of the peak without assuming a specific form of the regression function.

The goal of this paper is threefold. First, to propose the isotonic regression (Robertson et al.,1988) as a sensible tool that outperforms joinpoint regression in unimodal patterns. Second, to derive conditional tests for checking unimodality and for testing monotonicity against unimodality. Akaike Information criterion (AIC) measures are also provided and confidence intervals for the peaks are given using a parametric bootstrap approach. Third, to analyze trend changes and its location in breast cancer mortality.

Several authors have dealt with related problems using the isotonic regression for monotone regression models or for alternative shape constraints. Some representative references are Brunk (1970), Dykstra (1983), Hastie and Tibshirani (1986), Bachetti (1989), Huang (2002), Andersson et al. (2004), Meyer (2008), Shively et al. (2011) and Rueda and Lombardía (2012) among many others. There has been also considerable previous work on procedures testing homogeneity against monotonicity or unimodality (see for example Basso and Salmaso, 2011, Shi, 1988 and Wolfe, 2006), but the problem of considering testing monotonicity against unimodality has not been studied yet. This particular test will be very useful in the application considered in this paper. Although it is known that breast cancer mortality rates show a clear unimodality pattern in some developed countries (Malvezzi et al. 2012), we are particularly interested here in testing if this unimodality pattern persists in small areas within the country. The methodology derived will be also useful to analyze data from a variety of applications in many fields whenever the interest relies on checking if the functional relationship between the response and the explanatory variable exhibit a unimodal or monotone pattern.

The rest of the paper is laid out as follows. Section 2 introduces the isotonic regression and the AIC criterion to choose between monotonicity and unimodality. In Section 3 the condi- 
tional tests for checking unimodality and testing monotonicity against unimodality are derived. Section 4 illustrates the methodology analyzing breast cancer mortality data in Spain and its Autonomous Regions in the period 1975-2005. A comparison with the results obtained using joinpoint regression is also provided. A discussion closes this paper.

\section{Isotonic regression for fitting and testing temporal trends in mortality rates}

Let $Y_{t}$ be the number of deaths in year $t$, from $t=t_{1}, \ldots, t_{n}$ in a given region. It is commonly assumed that $Y_{t}$ is Poisson distributed with mean $\mu_{t}=n_{t} r_{t}$, where $r_{t}$ is the unknown rate of mortality and $n_{t}$ is the population at risk. Once the rate is estimated at each year we could represent the mortality temporal trend in that particular region. Our interest here relies on checking if there exists a break-point in the temporal trend, and if it does, to estimate it. In what follows a brief introduction of the underlying methodology is provided. Let $\mathbf{r}=\left(r_{t_{1}}, \ldots, r_{t_{n}}\right)$ be the vector of rates. Let $M=\left\{\mathbf{r} \in \Re^{n} \mid r_{t_{1}} \leq \cdots \leq r_{t_{n}}\right\}$ be the set representing the monotonicity of rates over the study period (this means that there is not a rate trend change over the years) and $U=\left\{\mathbf{r} \in \Re^{n} \mid r_{t_{1}} \leq \cdots \leq r_{q} \geq r_{q+1} \geq \cdots \geq r_{t_{n}}, t_{1} \leq q \leq t_{n}\right\}$ be the set representing a temporal pattern of unimodality. To check for the presence of a unimodality pattern and therefore, the existence of a break point in the rate temporal trend $\mathbf{r}$, we consider first the simple case where an initial guess for the mode or break point $(\psi), \psi_{0}=q$ is given. Consider the following hypotheses

$$
\begin{gathered}
H_{0 q}: r_{t_{1}} \leq \cdots \leq r_{q}=r_{q+1}=\cdots=r_{t_{n}}, \\
H_{1 q}: r_{t_{1}} \leq \cdots \leq r_{q} \geq r_{q+1} \geq \cdots \geq r_{t_{n}}, \\
H_{2}: \mathbf{r} \in \Re^{n} .
\end{gathered}
$$

Two tests will be performed. The first test checks for the existence of unimodality. Namely

$$
H_{1 q} \quad \text { vs. } \quad H_{2}-H_{1 q}
$$

and the second one checks for the presence of a break-point before the end of the period under study

$$
H_{0 q} \quad \text { vs. } \quad H_{1 q}-H_{0 q} .
$$

The maximum likelihood estimators $\widehat{\mathbf{r}}^{0 q}$ and $\widehat{\mathbf{r}}^{1 q}$ under $H_{0 q}$ and $H_{1 q}$ are obtained by solving

$$
\max _{\left\{\mathbf{r} \in \Re^{n} \mid r_{t_{1}} \leq \cdots \leq r_{q}=r_{q+1}=\cdots=r_{t_{n}}\right\}} l(\mathbf{r})
$$

and

$$
\max _{\left\{\mathbf{r} \in \Re^{n} \mid r_{t_{1}} \leq \cdots \leq r_{q} \geq r_{q+1} \geq \cdots \geq r_{t_{n}}\right\}} l(\mathbf{r})
$$


where $l(\mathbf{r})$ is the Poisson log-likelihood given by

$$
l(\mathbf{r})=\left(\sum_{t=t_{1}}^{t_{n}} y_{t} \log \left(r_{t}\right)+y_{t} \log \left(n_{t}\right)-\log \left(y_{t} !\right)-r_{t} n_{t}\right) .
$$

In the case of $\psi$ being unknown, a plug-in approach is performed. Firstly, the observed mode $\widehat{\psi}=q$ is derived by solving

$$
\max _{t_{1} \leq q \leq t_{n}}\left[\max _{\left\{\mathbf{r} \in \Re^{n} \mid r_{t_{1}} \leq \cdots \leq r_{q} \geq r_{q+1} \geq \cdots r_{t_{n}}\right\}} l(\mathbf{r})\right]
$$

and then, it is plugged in tests (1) and (2). In the case that (5) has multiple solutions, the highest value is selected as the mode. The solution to the optimization problems (3), (4) and (5) is achieved using isotonic regression. These problems can be solved as a weighted least squares fit of the observed rates $\mathbf{v}=\left(v_{t_{1}}, \ldots, v_{t_{n}}\right)$, where $v_{t}=y_{t} / n_{t}$ and the weights are given by $w_{t}=n_{t}$, subject to monotonicity or unimodality constraints. A convenient algorithm for carrying this out is stated by Shi (1988) and it has been implemented in the R package Iso (see Turner, 2013). Confidence intervals (CI) can be also computed. The methodology to derive CIs under restrictions has not been treated very extensively in the literature. Some interesting papers are written by Hwang and Peddada (1994), Peddada (1997) and Pan (1997). More recently, Strand et al. (2010) compare alternative proposals to derive CI for monotone regression pointing out the parametric bootstrap as the best choice. Parametric bootstrap has been considered in this paper to obtain CIs.

\subsection{The AIC criterion and the degrees of freedom}

To choose between a monotone $M$ or a unimodal $U$ pattern, an AIC-type criterion similar to the AIC criterion (Akaike, 1973) used in linear regression is proposed. This alternative is simpler than the test approach we will derive in the next section. A general definition of this criterion for a restricted statistical model with parameter space $\mathbf{r} \in K$, where $K$ is any subset in $\Re^{n}$ is given by $A I C\left(\widehat{\mathbf{r}}^{K}\right)=-2 l\left(\widehat{\mathbf{r}}^{K}\right)+2 g\left(D_{K}(\mathbf{v})\right), l($.$) is the log-likelihood of the estimated model, g\left(D_{K}(\mathbf{v})\right)$ is a penalty term, $g$ is a real function often defined as $g(x)=x$ and $D_{K}(\mathbf{v})$ are the model degrees of freedom, also called the divergence. They are generally defined as $D_{K}(\mathbf{v})=\sum_{t=t_{1}}^{t_{n}} \frac{\partial \widehat{r}^{K}(\mathbf{v})}{\partial v_{t}}$, and particularly here $D_{K}(\mathbf{v})=n-\sharp\left[t, \widehat{\mathbf{r}}_{t}^{K}=\widehat{\mathbf{r}}_{t+1}^{K}\right]$. The use of the AIC statistic in isotonic models backs to Anraku (1999), yet posterior modifications have been introduced (see Zhao and Peng, 2002, and Liu et al. 2009). In this paper, the idea of Kato (2009) and Rueda (2013) proposing a penalty term equal to $2 D_{K}(\mathbf{v})$ in a regression context has been applied. Although computing $D_{K}(\mathbf{v})$ is straightforward in most parametric models, the restricted case is not so simple (Meyer and Woodruff, 2000). In particular, $D_{K}(\mathbf{v})$ is a random quantity in this context (data-dependent). For the problem we are dealing with, the interesting AIC measures are given by using $K=U$, called AICU, and $K=M$, called AICM. 


\section{The conditional likelihood ratio test}

In this section the conditional tests for testing (1) and (2) are derived. Initially, normal distributions with known variances are assumed. The case of unknown variances and the Poisson model as well as other exponential distributions will be considered as extensions of this simple model. Let us assume that $Y_{t} \sim N\left(r_{t}, \sigma_{t}\right)$, where $\sigma_{t}$ are known parameters. Here, the maximum likelihood estimate (MLEs) of $\mathbf{r}$ under $H_{0 q}$ and $H_{1 q}$ are the solutions to the optimization problems (3) and (4) respectively, where now $l(\mathbf{r})$ is the normal $\log$-likelihood. The MLEs are also obtained through the isotonic regression of $\mathbf{v}=\left(v_{1}, \ldots, v_{t_{n}}\right)$, where $v_{t}=y_{t}$ and $w_{t}=1 / \sigma_{t}$.

Let us consider $H_{0}: \mathbf{r} \in K_{0}$ and $H_{a}: \mathbf{r} \in K_{a}$, where $K_{0}$ and $K_{a}$ are any subsets in $\Re^{n}$, and the testing problem $H_{0}$ vs. $H_{a}-H_{0}$. For this general testing problem the likelihood ratio statistic $T$ is defined as

$$
T=2\left(l\left(\hat{\mathbf{r}}^{a}\right)-l\left(\hat{\mathbf{r}}^{0}\right)\right),
$$

where $l($.$) is the normal log-likelihood and \hat{\mathbf{r}}^{a}$ and $\hat{\mathbf{r}}^{0}$ are the MLE under the alternative and the null hypothesis respectively. Let $D=D_{K_{a}}(\mathbf{v})-D_{K_{0}}(\mathbf{v})$ be the data-dependent degrees of freedom of $T$. Under suitable regularity conditions, the distribution of $T$ has proven to be conditionally distributed as a $\chi_{d}^{2}$ when $r_{t_{1}}=\cdots=r_{t_{n}}$ (in what follows we denote $\mathbf{r}_{0}$ a vector whose components are equal), and $d$ is the observed value of $D$, i.e,

$$
\operatorname{pr}_{\mathbf{r}_{0}}(T \leq c \mid D=d)=\operatorname{pr}\left(\chi_{d}^{2} \leq c\right),
$$

(see Menéndez et al., 1992 and $\mathrm{Hu}$ and Wright, 1994). The conditional $\alpha$ level test rejects when $T \geq c(d)$ where $c(d)$ is defined as the $1-\alpha^{\prime}$ percentile of the $\chi_{d}^{2}$ distribution such that

$$
\alpha^{\prime}=\operatorname{pr}\left(\chi_{d}^{2} \geq c(d)\right)=\frac{\alpha}{1-p r_{\mathbf{r}_{0}}(T=0)} .
$$

The parameter configuration $\mathbf{r}_{0}$ has proven to be the least favorable configuration (LFC) of parameters under the null hypothesis for the likelihood ratio test (LRT) in regular testing problems. Besides, it has been shown that, asymptotically, the LFC is also $\mathbf{r}_{0}$ for the conditional test under non oblique hypothesis (see Wollan and Dykstra, 1986 and Menéndez et al., 1991). This important fact guarantees that the conditional test is asymptotically an $\alpha$ level test allowing to obtain a $p$-value from a $\chi_{d}^{2}$ distribution. When $p r_{\mathbf{r}_{0}}(T=0)$ is a very small number for moderate or large $n, c(d)$ is simply defined as the $1-\alpha$ percentile of the $\chi_{d}^{2}$ distribution. The use of conditional tests is not new in order restricted inference (ORI) (see for example Bartholomew, 1961 and Iverson and Harp, 1987). The standard LRT, also called chi-bar test, involves weights depending on unknown parameters that are difficult to compute. However, the conditional test is computationally much simpler and it also benefits from an increase in power (see for example, Menéndez et al., 1991). The idea of using a conditional test on data-dependent degrees of freedom in the context of boundary constraints has been borrowed from ORI by Susko (2013) This paper highlights the advantages of this approach and illustrates its use in different settings. 
Table 1: $\operatorname{pr}\left(T_{M_{q}}=0\right)$ for different values of $n$ and $q$, equal weights and independent $N(0,1)$ distributions

\begin{tabular}{lllll}
$n-q$ & $n=10$ & $n=20$ & $n=30$ & $n=40$ \\
\hline 1 & 0.3328 & 0.3100 & 0.3051 & 0.3067 \\
2 & 0.1914 & 0.1706 & 0.1653 & 0.1661 \\
3 & 0.1365 & 0.1148 & 0.1139 & 0.1081 \\
4 & 0.1062 & 0.0834 & 0.0832 & 0.0746 \\
5 & 0.0910 & 0.0658 & 0.0662 & 0.0610 \\
6 & 0.0793 & 0.0528 & 0.0518 & 0.0490 \\
7 & 0.0823 & 0.0471 & 0.0480 & 0.0431 \\
8 & 0.0857 & 0.0437 & 0.0408 & 0.0384 \\
9 & 0.1055 & 0.0396 & 0.0378 & 0.0341 \\
10 & - & 0.0373 & 0.0317 & 0.0292 \\
11 & - & 0.0361 & 0.0315 & 0.0282 \\
12 & - & 0.0308 & 0.0250 & 0.0219 \\
13 & - & 0.0299 & 0.0240 & 0.0206 \\
14 & - & 0.0280 & 0.0213 & 0.0184 \\
15 & - & 0.0289 & 0.0204 & 0.0167 \\
16 & - & 0.0286 & 0.0198 & 0.0163 \\
17 & - & 0.0308 & 0.0199 & 0.0174 \\
18 & - & 0.0327 & 0.0170 & 0.0156 \\
19 & - & 0.0453 & 0.0167 & 0.0137 \\
20 & - & - & 0.0150 & 0.0151 \\
21 & - & - & 0.0151 & 0.0155 \\
22 & - & - & 0.0162 & 0.0141 \\
23 & - & - & 0.0170 & 0.0144 \\
24 & - & - & 0.0192 & 0.0122 \\
25 & - & - & 0.0170 & 0.0130 \\
26 & - & - & 0.0179 & 0.0121 \\
27 & - & - & 0.0203 & 0.0121 \\
28 & - & - & 0.0216 & 0.0117 \\
29 & - & - & 0.0293 & 0.0131 \\
30 & - & - & - & 0.0118 \\
\hline & & & &
\end{tabular}




\subsection{Testing unimodality against $\Re^{n}$}

In this section testing problem (1) is considered. Let $T_{1 q}$ be the LR statistic, the conditional test rejects $H_{1 q}$ when $T_{1 q} \geq c(d)$ and $c(d)$ is defined in (6). Problem (1) is regular because the alternative is a linear subspace. Therefore, following similar arguments to those given by Iverson and Harp (1987) it can be proved that the level of the test is attained at $\mathbf{r}_{0}$ and that the size of the test for other parameter values in the null hypothesis is asymptotically smaller than $\alpha$.

Remark 1 A small practical difficulty with the conditional test just defined is the evaluation of $p r_{\mathbf{r}_{0}}\left(T_{1 q}=0\right)$, which depends on $w_{t}$, $q$, and $n$. It can be computed by simulation. Moreover, taking into account that $\operatorname{pr}\left(\mathbf{v} \in\left\{r_{t_{1}} \leq \cdots \leq r_{q} \geq r_{q+1} \geq \cdots \geq r_{t_{n}}\right\}\right) \leq \operatorname{pr}(\mathbf{v} \in$ TO $)$, where TO is the tree order cone (see Robertson et al., 1988), it is easy to prove that $p_{\mathbf{r}_{0}}\left(T_{1 q}=0\right) \rightarrow_{n \rightarrow \infty} 0$. In particular, if $w_{t}=w, p_{\mathbf{r}_{0}}\left(T_{1 q}=0\right) \leq \frac{1}{(n-1) !}$. Therefore, to simplify computation, in scenarios with moderate or large $n, c(d)$ is simply defined as the $1-\alpha$ percentile of the $\chi_{d}^{2}$ distribution. The loss of power is not significant for moderate or large $n$ values.

Remark 2 i) There is a natural extension when working with normal distributions with unknown but common variances. The conditional test is easy to formulate using the results by Menéndez et al. (1992). The F distribution is then used instead of the chi-squared distribution for the test statistic.

ii) For the case of the Poisson distribution and other exponential families, the results above are true when $\left(n_{t}\right)$ is big enough. The fact that the asymptotic distribution of the LR statistic is the same as the one in the normal case (see Robertson et al., 1988; Chapter 4) guarantees that the results are asymptotically valid in exponential families.

\subsection{Testing monotonicity against unimodality}

In this section testing problem (2) is considered. The corresponding LR statistic is denoted by $T_{0 q}$. This is a non regular problem as both hypotheses are defined by cones that are not linear subspaces. However, we will show that also in this case the conditional test can be successfully applied. The conditional test rejects $H_{0 q}$ when $T_{0 q} \geq c(d)$, where $c(d)$ is defined as the $1-\alpha^{\prime}$

percentile of a $\chi_{d}^{2}$ distribution with $\alpha^{\prime}=\frac{\alpha}{1-p r_{\mathbf{r}_{0}}\left(T_{0 q}=0\right)}$. Denote by $\Pi_{q}(\mathbf{r})$ the size in $\mathbf{r}$ of the conditional test defined for testing problem (2). Then,

$$
\Pi_{q}(\mathbf{r})=\sum_{d} p r_{\mathbf{r}}\left(T_{0 q} \geq c(d) \mid D=d\right) p r_{\mathbf{r}}(D=d) .
$$

Theorem 3 proves two results. Firstly, we show that the distribution of the LR statistic $T_{0 q}$ conditioned to $D=d$ is a $\chi_{d}^{2}$ and subsequently, that the size of the conditional test for $\mathbf{r}_{0}$ equals $\alpha$. Moreover, it is also possible to prove that the size of the conditional test is smaller than $\alpha$ for other parameter values in the null hypothesis, using both simulation and asymptotic theoretical results (Menéndez et al., 1991).

Theorem 3 Let $T_{0 q}$ be the LR statistic for testing $H_{0 q}$ against $H_{1 q}-H_{0 q}$ and $\Pi_{q}$ the function defined in (7). Then, we have: i) $p r_{\mathbf{r}_{0}}\left(T_{0 q} \geq c \mid D=d\right)=p r\left(\chi_{d}^{2} \geq c\right)$ and ii $) \Pi_{q}\left(\mathbf{r}_{0}\right)=\alpha$. Proof of Theorem 3 is given in the Appendix. 
Remark 4 To use the test in practice, $p r_{\mathbf{r}_{\mathbf{0}}}\left(T_{0 q}=0\right)$ needs to be computed. For given $n$, $q$, and $w_{i}$ it can be easily obtained by simulation (see Tables 1 and 2).

Remark 5 There is a natural extension of these results when working with normal distributions with unknown but common variances. Moreover, results are also asymptotically valid in exponential families (Robertson et al., 1988, Chapter 4).

Table 2: $\operatorname{pr}_{W}\left(T_{M_{q}}=0\right)$ for $n=31$ and different values of $q$ and $w_{t}, 1 \leq t \leq 31$. Independent $N\left(0, s d_{t}\right), s d_{t}=1 / \sqrt{w_{t}}$ distributions

\begin{tabular}{lllll}
$n-q$ & $w_{t}=1$ & $w_{t}=t$ & $w_{t}=n-t+1$ & $w_{t}=n_{t}$ \\
\hline 1 & 0.3204 & 0.3260 & 0.3277 & 0.3131 \\
2 & 0.1657 & 0.1738 & 0.1427 & 0.1597 \\
3 & 0.1113 & 0.1194 & 0.0802 & 0.1089 \\
4 & 0.0832 & 0.0894 & 0.0507 & 0.0796 \\
5 & 0.0678 & 0.0746 & 0.0343 & 0.0656 \\
6 & 0.0556 & 0.0628 & 0.0262 & 0.0545 \\
7 & 0.0471 & 0.0544 & 0.0197 & 0.0464 \\
8 & 0.0390 & 0.0463 & 0.0157 & 0.0391 \\
9 & 0.0368 & 0.0432 & 0.0136 & 0.0370 \\
10 & 0.0347 & 0.0387 & 0.0119 & 0.0341 \\
11 & 0.0295 & 0.0352 & 0.0073 & 0.0299 \\
12 & 0.0259 & 0.0329 & 0.0076 & 0.0263 \\
13 & 0.0249 & 0.0299 & 0.0084 & 0.0247 \\
14 & 0.0236 & 0.0292 & 0.0070 & 0.0238 \\
15 & 0.0214 & 0.0262 & 0.0055 & 0.0224 \\
16 & 0.0208 & 0.0256 & 0.0051 & 0.0208 \\
17 & 0.0183 & 0.0237 & 0.0052 & 0.0188 \\
18 & 0.0187 & 0.0232 & 0.0055 & 0.0189 \\
19 & 0.0185 & 0.0237 & 0.0052 & 0.0191 \\
20 & 0.0177 & 0.0206 & 0.0044 & 0.0181 \\
21 & 0.0156 & 0.0198 & 0.0046 & 0.0163 \\
22 & 0.0160 & 0.0177 & 0.0052 & 0.0162 \\
23 & 0.0165 & 0.0175 & 0.0058 & 0.0171 \\
24 & 0.0157 & 0.0157 & 0.0060 & 0.0161 \\
25 & 0.0157 & 0.0153 & 0.0053 & 0.0153 \\
26 & 0.0159 & 0.0142 & 0.0055 & 0.0161 \\
27 & 0.0180 & 0.0127 & 0.0069 & 0.0178 \\
28 & 0.0196 & 0.0112 & 0.0076 & 0.0198 \\
29 & 0.0239 & 0.0121 & 0.0105 & 0.0239 \\
30 & 0.0338 & 0.0145 & 0.0155 & 0.0347 \\
\hline & & & &
\end{tabular}




\section{Illustration}

The test derived in Section 3 is illustrated using female breast cancer mortality data from Spain in the period 1975-2005. It is known (see Malvezzi et al., 2012) that in many developed countries like Spain, breast cancer mortality increased sharply until the nineties and then decreased. In this section we are interested in testing if this unimodality pattern persists in all the Autonomous Regions of Spain. An Autonomous Region is constituted by one single or a group of provinces and it has its own local government. The problem of checking unimodality in Autonomous Regions is of interest as they have their own health system and habits and therefore some differences may be found. Isotonic regression will be also used to find break points and confidence intervals for the regions where unimodality persists. However, the common procedure to estimate break points in epidemiology is the well-known joinpoint regression model (see for example, Statistical Research and Applications Branch, National Cancer Institute, 2009), therefore, a comparison between isotonic regression and joinpoint regression will be made first, to show the reasons why isotonic regression will be used in this context. The comparison will be made analyzing both real data and simulated data under the scenario of unimodality pattern (a single break point) mimicking the real mortality data from Spain. A similar procedure is used by Turner and Wollan (1997). These authors demonstrate that the isotonic estimator of the mode is consistent, simple to understand, and performs satisfactorily in practice. In the following we show that the isotonic estimator is working well in the epidemiological context considered here. However, the joinpoint regression method has two serious drawbacks; it could be seriously biased and it is not robust because when we slightly modify the studied period, the estimator provides a different mode, in contrast to the isotonic estimator.

\subsection{Comparing isotonic regression and joinpoint regression}

When studying trends in mortality or incidence data, it is often of interest to uncover break points. The well-known joinpoint regression models, also called segmented regression models, have been used for this aim. These models explain the relationship between the response and the explanatory variables by means of different lines called segments. In this particular application we know in advance the existence of a single break point. In this regard, Ugarte et al. (2010) report a sharp increase of female breast cancer mortality and then a downturn around the nineties in different age-groups $(<45,45-64, \geq 65)$. The epidemiological literature also reported that the decline of breast cancer mortality in Spain is delayed when the age of the group is increased. The decline starts earlier in younger women probably reflecting the increasing survival of cases (Berrino et al., 2007). Joinpoint regression finds the break point using the following model in each age-group

$$
\begin{aligned}
y_{t} & \sim \operatorname{Poisson}\left(r_{t} n_{t} / 100000\right) \\
\log \left(y_{t}\right) & =\log \left(n_{t} / 100000\right)+\beta_{0}+\beta_{1} t+\beta_{2}(t-\psi)_{+}
\end{aligned}
$$

where $y_{t}$ is the female mortality counts from breast cancer in a specific age-group at time $t, n_{t}$ is the population at risk and $r_{t}$ is the corresponding mortality rate (per 100,000 individuals). According to such parametrization, $\beta_{0}$ and $\beta_{1}$ are the intercept and the slope respectively of a 
line adjusted up to the break-point $\psi$ and $\beta_{2}$ is the difference-in-slopes. The nonlinear term in (8) has an approximate intrinsic linear representation permitting to express the model as a kind of generalized linear model (Muggeo, 2003). Given an initial guess for the break point, $\tilde{\psi}$, model (8) can be estimated by fitting iteratively the generalized linear model with linear predictor

$$
\log \left(n_{t} / 100000\right)+\beta_{0}+\beta_{1} t+\beta_{2}(t-\tilde{\psi})_{+}+\gamma I(t>\tilde{\psi})^{-}
$$

where $I(\cdot)^{-}=-I(\cdot)$ and $\gamma$ is the parameter that can be understood as a re-parametrization of $\psi$ accounting for the break point estimation. The R package segmented by Muggeo (2008) offers facilities to estimate and summarize generalized linear models with segmented relationships. It uses a method that allows to estimate simultaneously all the model parameters yielding also, at the possible convergence, the approximate full covariance matrix. In our application we have fitted the model using this package.

Table 3: Break point estimates of breast cancer mortality rates $\hat{\psi}$ as well as confidence intervals by age groups using both joinpoint and isotonic regression

\begin{tabular}{lccc}
\hline Joinpoint & $\hat{\psi}$ & $\mathrm{SE}(\hat{\psi})$ & $90 \%$ CI \\
\hline$<45$ years & 1993 & 0.805 & {$[1991,1994]$} \\
45-64 years & 1993 & 0.281 & {$[1992,1993]$} \\
$\geq 65$ years & 1992 & 0.347 & {$[1992,1993]$} \\
\hline Isotonic & $\hat{\psi}$ & $\mathrm{SE}(\hat{\psi})$ & $90 \% \mathrm{CI}$ \\
\hline$<45$ years & 1991 & 1.644 & {$[1989,1995]$} \\
45-64 years & 1993 & 0.978 & {$[1991,1995]$} \\
$\geq 65$ years & 1995 & 1.146 & {$[1993,1997]$} \\
\hline
\end{tabular}

Table 3 shows estimated break points, standard errors, and confidence intervals $(1-\alpha=0.9)$ of the break points computed using joinpoint and isotonic regression. Surprisingly, in the joinpoint regression the same break point is found for the first two age-groups whereas for the third age group the break point is estimated one year in advance, something unexpected by epidemiologists. However, isotonic regression provides more reasonable and comprehensive results. As reported in the epidemiological literature, the mortality decline starts earlier in the first (younger) age-group and from there on it suffers a delay. Computational code to fit both models are available from the authors under request.

\subsubsection{Simulation study}

To compare joinpoint regression and isotonic regression more formally, we made a small simulation study in the scenario of the Spanish breast cancer mortality rates. We first create a new data set simulating data from a Poisson distribution using the same population at risk as in our original data set (see Turner and Wollan, 1997). We then smooth the data using a generalized additive model with the mortality counts as response, the log of the population per 100,000 women as an 
offset, and a smooth function of the time approximated using P-splines with B-spline bases. The estimated smooth rates were considered the "true rates". The real value of the break point is considered to be the mode of the smoothed curve, more specifically the nearest integer was selected (see the first row of 1). Finally, the simulation was run following the next steps. 1) Generate 1000 samples of size $n$ in each age-group from a Poisson distribution with mean $r_{t}=n_{t} / 100000 * \hat{r}_{t}$, where $n_{t}$ is the population at risk in year $t$, and $\hat{r}_{t}$ are the estimated smooth rates. 2) Fit both a joinpoint regression and a isotonic regression to each simulated data set and obtain break points. 3) Compute the mean bias (MB) and the root mean squared error (RMSE) of the break point estimators. $M B=\frac{1}{1000} \sum_{j=1}^{1000}\left(\hat{\psi}_{j}-\psi\right), \quad R M S E=\sqrt{\frac{1}{1000} \sum_{j=1}^{1000}\left(\hat{\psi}_{j}-\psi\right)^{2}}$ where $\hat{\psi}_{j}$ is the break point estimate using the $j^{\text {th }}$ simulated sample for each age-group and $\psi$ is the true value of the break point for that age-group. Results of the simulation study are given in Table 4. Graphical representation of the results are shown in the second (joinpoint regression) and third row (isotonic regression) of 1 . Both, Table 4 and the graphical results point out a much larger bias for the joinpoint regression analysis. In this particular simulation study we find larger RMSE in two age groups for the joinpoint analysis. It is striking that coverage probabilities even reach the zero value for particular unimodal patterns (like the simulated pattern of the third age-group). Consequently, jointpoint regression should not be used when the underlying shape of the curve is not piecewise linear. The reason for the over-coverage in the isotonic case is due to the discrete nature of the mode distribution.

We have also analyzed breast cancer mortality rates (real data) for two more periods: 1975-2010 and 1980-2010. When representing crude rates a unimodal pattern is observed too. However, while the isotonic regression model provides the same break points in all the periods, the joinpoint regression model gives different break point estimates depending on the period. In this sense, we may affirm that the isotonic regression model is robust.

Table 4: MB, RMSE, and coverage probabilities using joinpoint and isotonic regression. The first column gives the true break point $\psi$ in each age-group

\begin{tabular}{|c|c|c|c|c|c|}
\hline \multicolumn{6}{|c|}{ Joinpoint } \\
\hline & $\psi$ & MB & RMSE & Coverage $90 \%$ & Coverage $95 \%$ \\
\hline$<45$ years & 1990 & 1.503 & 1.749 & 62.4 & 72.5 \\
\hline 45-64 years & 1991 & 1.127 & 1.213 & 35.6 & 40.0 \\
\hline$\geq 65$ years & 1996 & -4.475 & 4.540 & 0.0 & 0.0 \\
\hline \multicolumn{6}{|c|}{ Isotonic } \\
\hline & $\psi$ & MB & RMSE & Coverage $90 \%$ & Coverage $95 \%$ \\
\hline$<45$ years & 1990 & 0.577 & 1.727 & 97.9 & 99.6 \\
\hline 45-64 years & 1991 & 0.268 & 1.164 & 97.9 & 99.3 \\
\hline$\geq 65$ years & 1996 & 0.544 & 2.317 & 96.5 & 98.8 \\
\hline
\end{tabular}



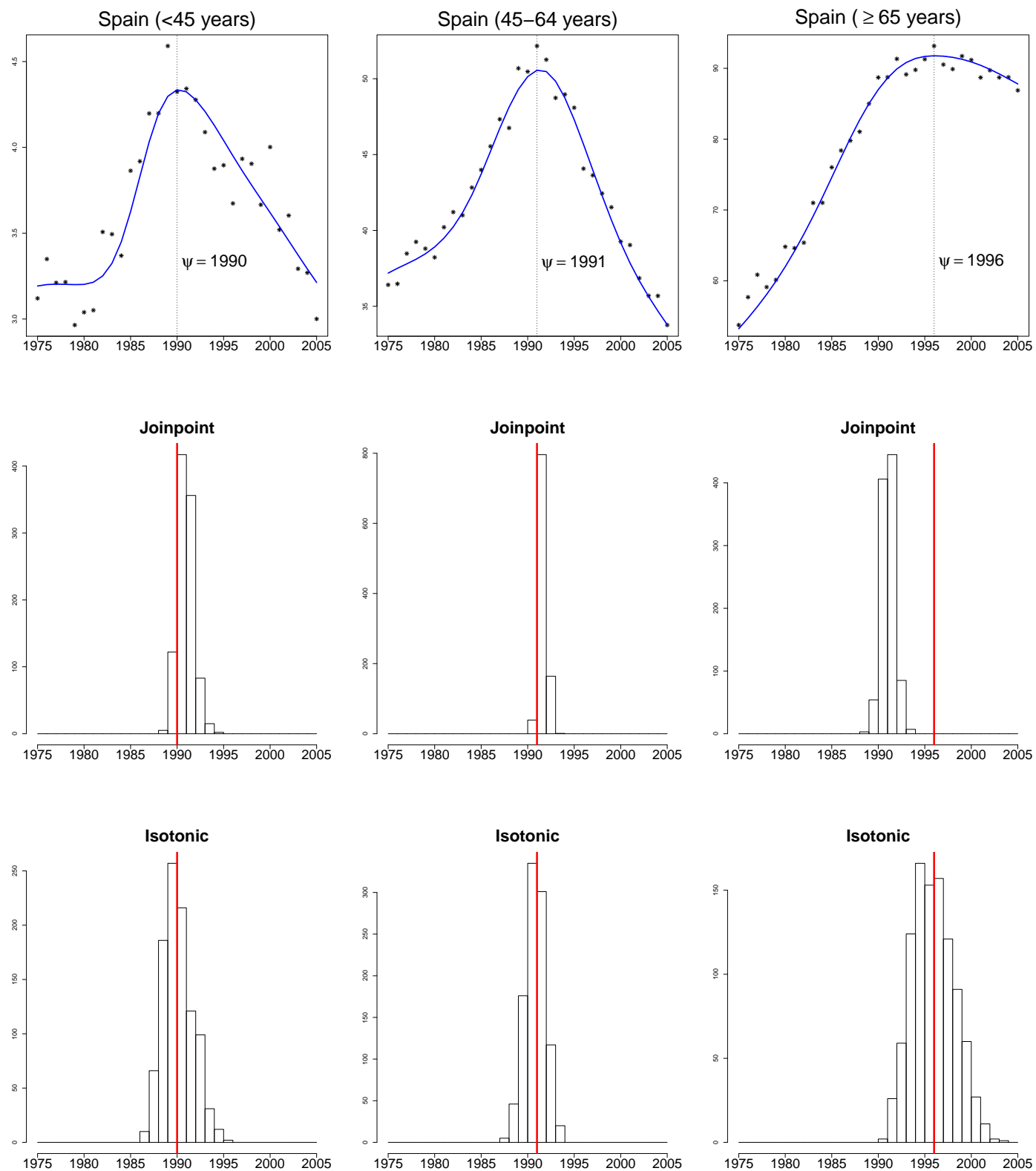

Figure 1: In the first row, the true models for breast cancer mortality rates in the three agegroups. The crude rates have been smooth using a gam model. The true break points are also represented. In the second row, histograms of the estimated break points using joinpoint regression. The third row shows histograms of estimated break points using isotonic regression 


\subsection{Checking unimodality in Spanish regions}

The interest relies on testing if the unimodality pattern observed in the 45-65 age-group in the whole of Spain is also observed by Autonomous Regions. In those regions where unimodality is present, the break point estimate will be computed using isotonic regression. As already stated, this is important as each Spanish region has its own health system, and then, we might expect to observe different break point estimates among regions. In particular, Spanish regions have implemented breast cancer screening programs in different moments and this could affect the mortality decline differently. A test for checking unimodality versus any other pattern is performed first. Results are given in column $p$-values of 5 . The null hypothesis is only rejected for Castilla and León. Then, the test developed in Section 3 for checking monotonicity against unimodality is calculated for the rest of the regions. The null hypothesis is rejected in all the cases and then, a unimodality pattern is considered ( $p$-valuesMU in 5). We have carefully analyzed the data of Castilla and León in this period. It seems there could be a problem registering the data in 1995. There were 111 mortality cases in 1994 and the same number of cases in 1996, whereas in 1995 the number of cases increased till 165, what is a bit surprising. With a moderate change in this data, the unimodality pattern is accepted. Moreover, using the AIC criteria (see Table 5 where $A I C U=61.09, A I C M=116.99$ ), the unimodal pattern seems to be preferable to the monotone model also in Castilla and León. As expected, different break point estimates in the regions are observed. Whereas there are regions where the downturn starts in the mid-eighties like Aragón, Asturias, Extremadura, Navarra, and País Vasco, there are other regions where the decline in breast cancer mortality is delayed till mid-nineties like Castilla-La Mancha, Andalucía, Balear Islands, Galicia or Madrid.

\section{Discussion}

To look for change points when analyzing cancer mortality trends is crucial for epidemiologists and public health researchers. A decreasing trend change could mean for example disease treatment improvements or the effect of a screening program. This paper presents a new methodology to test for a break point in the presence of a unimodal trend. In particular, two alternative procedures have been derived: a conditional test for checking monotonicity against unimodality and a simpler approach based on an AIC criterion. In addition, using isotonic regression we are also able to locate the break point and to derive the corresponding confidence interval by means of parametric bootstrap. The traditional method for finding trend changes and their location in the epidemiological field is joinpoint regression. However, we show in this paper that this method could fail even in the case of finding a single break point if the true shape of the mortality trend is not piecewise linear, as in the real case analyzed here. Moreover, the method is not robust because when we slightly modify the studied period the breakpoint changes, whereas isotonic regression is robust in this sense. It is important to point out the simplicity of the provided conditional tests using the chi-square distribution and the data-dependent degrees of freedom. It can be broadly applied to analyze data when the interest relies on locating a peak or testing for a monotone increasing pattern. The gain in power when the alternative is reduced can be important in some cases, specifically when analyzing break point detection. The proposed test is derived under Poisson assumptions for the application used in this paper but it 
Table 5: Break point estimates using isotonic regression for breast cancer mortality rates (45-64 years) in all the Spanish Autonomous Regions, standard errors, confidence intervals, Akaike information criterion for the Monotone model (AICM) and Akaike information criterion for the Unimodal Model (AICU)

\begin{tabular}{|c|c|c|c|c|c|c|c|c|}
\hline & & $\hat{\psi}$ & $\operatorname{SE}(\hat{\psi})$ & $90 \% \mathrm{CI}$ & $p$-values & $p$-valuesMU & AICM & $\mathrm{AICU}$ \\
\hline 1 & Andalucía & 1994 & 1.143 & [1992，1995] & 0.995 & $8.966 \mathrm{e}-07$ & 68.16 & 41.11 \\
\hline 2 & Aragón & 1986 & 1.764 & {$[1986,1991]$} & 0.452 & $5.299 \mathrm{e}-08$ & 75.68 & 42.09 \\
\hline 3 & Asturias, Principado de & 1985 & 3.760 & {$[1985,1995]$} & 0.942 & $2.854 \mathrm{e}-04$ & 41.39 & 28.20 \\
\hline 4 & Balears, Illes & 1994 & 2.943 & {$[1986,1995]$} & 0.422 & $5.592 \mathrm{e}-08$ & 75.80 & 42.58 \\
\hline 5 & Canarias & 1989 & 2.360 & {$[1986,1995]$} & 0.531 & $1.752 \mathrm{e}-13$ & 101.39 & 40.87 \\
\hline 6 & Cantabria & 1989 & 3.756 & {$[1981,1995]$} & 0.639 & $8.887 \mathrm{e}-03$ & 42.36 & 37.30 \\
\hline 7 & Castilla - La Mancha & 1995 & 2.212 & {$[1989,1996]$} & 0.180 & $1.125 \mathrm{e}-03$ & 58.46 & 48.43 \\
\hline 8 & Castilla - León & 1991 & 1.235 & {$[1989,1996]$} & 0.019 & $4.192 \mathrm{e}-12$ & 116.99 & 61.09 \\
\hline 9 & Cataluña & 1993 & 1.780 & {$[1988,1994]$} & 0.977 & $0.000 \mathrm{e}+00$ & 233.89 & 38.13 \\
\hline 10 & Comunitat Valenciana & 1993 & 1.043 & {$[1990,1993]$} & 0.680 & $8.695 \mathrm{e}-13$ & 105.94 & 46.16 \\
\hline 11 & Extremadura & 1983 & 3.346 & {$[1983,1993]$} & 0.202 & $2.819 \mathrm{e}-03$ & 55.27 & 47.85 \\
\hline 12 & Galicia & 1994 & 2.022 & {$[1988,1994]$} & 0.490 & $1.327 \mathrm{e}-05$ & 66.00 & 45.47 \\
\hline 13 & Madrid & 1994 & 1.712 & {$[1989,1995]$} & 0.784 & $9.133 \mathrm{e}-14$ & 103.99 & 41.40 \\
\hline 14 & Murcia & 1991 & 3.168 & {$[1987,1997]$} & 0.928 & $4.164 \mathrm{e}-02$ & 37.87 & 37.35 \\
\hline 15 & Navarra & 1986 & 3.511 & {$[1986,1995]$} & 0.150 & $1.001 \mathrm{e}-06$ & 74.98 & 48.51 \\
\hline 16 & País Vasco & 1988 & 2.012 & {$[1987,1993]$} & 0.996 & $1.780 \mathrm{e}-10$ & 80.16 & 33.34 \\
\hline 17 & Rioja, La & 1993 & 1.637 & {$[1989,1995]$} & 0.232 & $3.745 \mathrm{e}-04$ & 59.77 & 47.11 \\
\hline
\end{tabular}

can be extended for general exponential models where the normal model is a particular case. The methodology developed in this paper could be also useful for a variety of applications other than the epidemiological application considered here. In particular, it could be useful for a better understanding of the nature of an epidemic. The $p$-values of the conditional test could be used for an early detection of the epidemic peak. An additional interesting application is the analysis of toxicological data. The basic principle of toxicology stating that the larger the dose of a chemical substance, the greater the response in the human body, is changing. In fact, some researchers have published studies where different non-linear relationships between dose and response, such as non-monotonicity, can possibly occur, implying that a more significant reaction could occur at a low dose as compared to the reaction observed at higher doses (see Vandenberg et al., 2012; Lim et al., 2013; Lagarde et al., 2015). This hypothesis needs to be scientifically verified with the appropriate statistical tools as it could highly impact the regulatory approaches for evaluating toxicity. Ecologists and environmental scientists are also often interested in determining whether a response achieves a maximum at an intermediate value of some explanatory variable. The work by Thomas (2010) documents a unimodal relationship between photosynthetic capacity and stem diameter. Neuroscientists are interested as well in the estimation of peaks in unimodal patterns (see O'Connell et al., 2012). 


\section{Appendix}

The mathematical tools for understanding the proofs included in this appendix are not basic. For those readers who are not expert, we recommend first to read carefully the classical book by Robertson et al. (1988). New notation is also used to simplify the proofs. Let us define $U$ as the union of cones $U_{q}$ such that $U=\cup_{q=t_{1}}^{t_{n}} U_{q}$, where $U_{q}=\left\{\mathbf{r} \in \Re^{n} \mid r_{t_{1}} \leq \cdots \leq r_{q} \geq r_{q+1} \geq\right.$ $\left.\cdots \geq r_{t_{n}}\right\}$. Then, the testing problems (1) and (2) can be reformulated as follows

$$
\begin{gathered}
H_{1 q}: \mathbf{r} \in U_{q} \quad \text { vs. } \quad H_{2}-H_{1 q}, \\
H_{0 q}: \mathbf{r} \in M_{q} \quad \text { vs. } \quad H_{1 q}-H_{0 q},
\end{gathered}
$$

where $M_{q}=M \cap U_{q} . R_{d}$ is referred as the set $D=d$. The estimators $\widehat{\mathbf{r}}^{1 q}$ and $\widehat{\mathbf{r}}^{0 q}$ are given by the weighted projection of $\mathbf{v}$ with weights $W=\operatorname{diag}\left(w_{t_{1}}, \ldots, w_{t_{n}}\right)$ in the convex cones $U_{q}$ and $M_{q}$, defined as $P_{W}\left(\mathbf{v} \mid U_{q}\right)$ and $P_{W}\left(\mathbf{v} \mid M_{q}\right)$ respectively (see Robertson et al., 1988). In order to simplify notation the weight matrix is eliminated from the projection operator in what follows. Lemma 1 shows that the cones defining the null and the alternative hypotheses in (11) verify a non oblique property. Then, Lemma 1 is used in the proof of Theorem 3 .

\subsection{Lemma 1}

(i) Let $L_{U}$ and $L_{M}$ be subsets verifying $P\left(\mathbf{v} \mid U_{q}\right)=P\left(\mathbf{v} \mid L_{U}\right), P\left(P\left(\mathbf{v} \mid U_{q}\right) \mid M_{q}\right)=P\left(\mathbf{v} \mid L_{M}\right)$. Then, $L_{M} \subset L_{U}$

(ii) The regions $M_{q}=M \cap U_{q}$ and $U_{q}$ are non oblique

$$
P\left(P\left(\mathbf{v} \mid U_{q}\right) \mid M_{q}\right)=P\left(\mathbf{v} \mid M_{q}\right) .
$$

\subsection{Proof of Lemma 1}

(i) $L_{U}$ and $L_{M}$ can be defined by a set of linear inequalities as follows:

$L_{M}=\left\{\mathbf{v} \in \mathbb{R}^{n} \mid v_{i}=v_{i+1}, i \in B\right\}$ and $\mathrm{七}_{U}=\left\{\mathbf{v} \in \mathbb{R}^{n} / v_{i}=v_{i+1}, i \in A\right\}$, where $B, A \subset\{1, \ldots, n-$ 1\}. The results will follow by showing that $A \subset B$. Let $i \in A$, then $P\left(\mathbf{v} \mid U_{q}\right)_{i}=P\left(\mathbf{v} \mid U_{q}\right)_{i+1}$. Now, as the cone $M_{q}$ is an acute cone we have that $P\left(P\left(\mathbf{v} \mid U_{q}\right) \mid M_{q}\right)_{i}=P\left(P\left(\mathbf{v} \mid U_{q}\right) \mid M_{q}\right)_{i+1}$ (see Menéndez and Salvador, 1991) and the result follows.

(ii) Let $L_{U}$ and $L_{M}$ be subspaces verifying $P\left(\mathbf{v} \mid U_{q}\right)=P\left(\mathbf{v} \mid L_{U}\right), P\left(P\left(\mathbf{v} \mid U_{q}\right) \mid M_{q}\right)=P\left(\mathbf{v} \mid L_{M}\right)$. Then, from basic properties of projections onto subspaces and convex cones, we have that for a given $\mathbf{v}$

$$
\mathbf{v}=P\left(\mathbf{v} \mid U_{q}\right)+P\left(\mathbf{v} \mid U_{q}^{p}\right)=P\left(\mathbf{v}\left|U_{q}\right| M_{q}\right)+P\left(\mathbf{v}\left|U_{q}\right| M_{q}{ }^{p}\right)+P\left(\mathbf{v} \mid U_{q}^{p}\right),
$$

from (i) we have that $L_{U} \subset L_{M}$, and then,

$$
\mathbf{v}=p\left(\mathbf{v} \mid L_{U}\right)+P\left(\mathbf{v} \mid L_{U}^{\perp}\right)=P\left(\mathbf{v} \mid L_{M}\right)+P\left(\mathbf{v} \mid L_{M}^{\perp} \cap L_{U}\right)+P\left(\mathbf{v} \mid L_{U}^{\perp}\right) .
$$


Now, as $M_{q} \subset U_{q}$, and both are closed convex cones, then $U_{q}{ }^{p} \subset M_{q}{ }^{p}$ and

$$
P\left(\mathbf{v}\left|U_{q}\right| M_{q}^{p}\right)+P\left(\mathbf{v} \mid U_{q}^{p}\right)=P\left(\mathbf{v} \mid L_{M}^{\perp} \cap L_{U}\right)+P\left(\mathbf{v} \mid L_{U}^{\perp}\right) \epsilon M_{q}^{p} .
$$

We also have that

$$
P\left(\mathbf{v}\left|U_{q}\right| M_{q}\right)=P\left(\mathbf{v} \mid L_{M}\right) \epsilon M_{q},
$$

and

$$
<P\left(\mathbf{v} \mid L_{M}\right), P\left(\mathbf{v} \mid L_{M}^{\perp} \cap L_{U}\right)+P\left(\mathbf{v} \mid L_{U}^{\perp}\right)>\leq 0 .
$$

Then, from statements above and basic properties of projections onto convex cones, we have that

$$
P\left(\mathbf{v} \mid L_{M}\right)=P\left(\mathbf{v} \mid M_{q}\right) .
$$

Now, for $\mathbf{v} \in \Re^{n}$ and $L_{U}$ and $L_{M}$ subspaces as in (i), we have that

$$
P\left(P\left(\mathbf{v} \mid U_{q}\right) \mid M_{q}\right)=P\left(\mathbf{v} \mid L_{M}\right)=P\left(\mathbf{v} \mid M_{q}\right)
$$

and this last statement shows that $M_{q}$ and $U_{q}$ are non-oblique.

\subsection{Proof of Theorem 3}

(i) From Lemma 1 (ii), Lemma 2.2. in Menéndez et al. (1992), and basic properties of polar cones, $T_{0 q}$ is given by

$$
T_{0 q}=\left\|P\left(\mathbf{v} \mid U_{q} \cap M_{q}^{p}\right)\right\|^{2}=\left\|P\left(\mathbf{v} \mid U_{q} \cap M^{p}\right)\right\|^{2} .
$$

Let $R_{d}$ be given by $R_{d}=\left\{\mathbf{v} \in \Re^{n} \mid P\left(\mathbf{v} \mid U_{q} \cap M^{p}\right)=P(\mathbf{v} \mid L)\right.$, $\left.\operatorname{dim} L=d\right\}$. From Lemma 1 is easy to prove that $R_{d}=\left\{\mathbf{v} \in \Re^{n} \mid P\left(\mathbf{v} \mid U_{q}\right)=P\left(\mathbf{v} \mid L_{U}\right), P\left(\mathbf{v} \mid M_{q}\right)=P\left(\mathbf{v} \mid L_{M}\right), d=\operatorname{dim}\left(L_{U}\right)-\operatorname{dim}\left(L_{M}\right)\right\}$. Now Shapiro(1988) shows that, for $\mathbf{r}_{0}$, the conditional distribution of $T_{0 q}$ given to the subsets $R_{d}$ is a chi-squared with $d$ degrees of freedom and the result follows.

(ii) From (i) and equality

$$
R_{0}=\left\{\mathbf{v} \in \Re^{n} \mid P\left(\mathbf{v} \mid U_{q}\right)=P\left(\mathbf{v} \mid M_{q}\right)\right\}=\left\{\mathbf{v} \in \Re^{n} / T_{0 q}(\mathbf{v})=0\right\},
$$

we have that

$$
\begin{array}{r}
\Pi_{q}\left(\mathbf{r}_{0}\right)=\sum_{d} p r_{r_{0}}\left(T_{0 q} \geq c(d) \mid \mathbf{v} \in R_{d}\right) p r_{r_{0}}\left(\mathbf{v} \in R_{d}\right)= \\
\sum_{d} p r\left(\chi_{d}^{2}>c(d)\right) p r_{r_{0}}\left(\mathbf{v} \in R_{d}\right)= \\
\frac{\alpha}{1-p r_{r_{0}}\left(T_{0 q}=0\right)} \sum_{d} p r_{r_{0}}\left(\mathbf{v} \in R_{d}\right)= \\
\frac{\alpha}{1-p r_{r_{0}}\left(T_{0 q}=0\right)}\left(1-p r_{r_{0}}\left(\mathbf{v} \in R_{0}\right)\right)=\alpha
\end{array}
$$

and the results follow. 
Acknowledgments: This work has been supported by the Spanish Ministry of Science and Innovation (project MTM 2011-22664 jointly sponsored with Feder grants, project MTM 201237129 and project MTM2014-51992-R). The work has been also partially supported by the Health Department of Navarre Government (Project 113, Res. 2186/2014).

\section{Bibliography}

Andersson, E., Bock, D. and Frisén M. (2004). Detection of truning points in business cycles. Journal of Business Cycle Measurement and Analysis, 1, 93-108.

Anraku, K. (1999). An information criterion for parameters under a simple order restriction. Biometrika, 86, 141-152.

Akaike, H. (1973). Information theory and the extension of the maximum likelihood principle. In: Petrov, B. N., Csaki, F. (Eds), Proceedings of the Second International Symposium on Information Theory. Akademiai Kiado, Budapest, 267-281.

Bachettti, P. (1989). Additive isotonic models. Journal of the American Statistical Association, 84, 289-294.

Banerjee, M., Mukherjee, D. and Mishra, S. (2009). Semiparametric binary regression models under shape constraints with an application to Indian schooling data. Journal of Econometrics, 149, 101-117.

Bartholomew, D.J. (1961). A test of homogeneity for means under restricted alternatives. Journal of the Royal Statistical Society, Series B, 23, 239-281.

Basso D., and Salmaso L. (2011). A permutation test for umbrella alternatives. Statistics and Computing 21, 45-54 (correction: 2001;20:655).

Berrino, F., De Angelis, R., Sant, M., Rosso, S., Lasota, M.B., Coebergh, J.W., et al. (2007). Survival for eight major cancers and all cancers combined for European adults diagnosed in 1995-99: results of the EUROCARE-4 study. Lancet Oncology, 8, 773-783.

Bock, D., Andersson, E. and Frisén, M. (2008). Statistical Surveillance of Epidemics: Peak Detection of Influenza in Swewden. Biometrical Journal, 1, 71-85.

Brunk, H.D. (1970). Estimation of isotonic regression (with discussion). In: Puri, M.L. (Ed). Nonparametric Techniques in Statistical Inference. Cambridge University Press, Cambridge.

De Souza, D. L B, Curado, M.P., Bernal, M.M. et al. (2013). Mortality trends and prediction of HPV-related cancers in Brazil. European Journal of Cancer Prevention, 22, 380-387.

Dykstra, R. (1983). An algorithm for restricted least squares regression. Annals of Statistics, 3, 401-421. 
Feder, P.I. (1975). On Asymptotic Distribution Theory in Segmented Regression Problems: Identified Case. Annals of Statistics, 3, 76-83.

Gunn, L.H. and Dunson, D.B. (2005). A transformation approach for incorporating monotone or unimodal constraints. Biostatistics, 6, 434-449.

Hastie, T. and Tibshirani, R. (1986). Generalized additive models. Statistical Science, 3, 297-310.

Huang, J. (2002). A note on estimating a partly linear model under monotonicity constraints. Journal of Statistical Planning and Inference, 107, 345-351.

Hudson, D. (1966). Fitting segmented curves whose join points have to be estimated. Journal of the American Statistical Association, 61, 1097-1129.

Hu, X. and Wright, F. T. (1994). Likelihood ratio tests for a class of non-oblique hypotheses. Annals of the Institute of Statistical Mathematics, 46(1), 137-145.

Hur, C., Miller, M., Kong, C.Y. et al. (2013). Trends in esophageal adenocarcinoma incidence and mortality. Cancer, 119(6), 1149-1158.

Hwang, J. G. and Peddada, S. D. (1994). Confidence interval estimation subject to order restrictions. Annals of Statistics, 22, 67-93.

Iverson, G.J. and Harp, S.A. (1987). A conditional likelihood ratio test for order restrictions in exponential families. Mathematical Social Sciences, 14, 141-159.

Kato, K. (2009). On the degrees of freedom in shrinkage estimation. Journal of Multivariate Analysis, 100, 1338-1352.

Kim, H.J., Fay, M.P., Feuer, E.J. and Midthune, D.N. (2000). Permutation tests for joinpoint regression with applications to cancer rates. Statistics in Medicine, 19, 335-351 (correction: $2001,20,655)$.

Köllmann, C., Bornkamp, B. and Ickstadt, K. (2012). Unimodal regression using BernsteinSchoenberg-splines and penalties. Technical Report. Universiy of Dortmund.

Lagarde, A., Beausoleil, C., Belcher, S.M., Belzunces, L.P., Emond, C., Guerbet, M. and Rousselle, C. (2015) Non-monotonic dose-response relationships and endocrine disruptors: a qualitative method of assessment. Environmental Health, 14, doi:10.1186/1476-069X$14-13$.

Lerman, P.M. (1980). Fitting Segmented Regression Models by Grid Search. Applied Statistics, 29, $77-84$.

Lim, C., Shen, P.K. and Peddada, S.D. (2013). Robust analysis of high throughput screening (HTS) assay data. Tecnometrics, 55, 150-160. doi:10.1080/00401706.2012.749166. 
Liu T, Lin N, Shi N, and Zhang B. (2009). Information criterion-based clustering with orderrestricted candidate profiles in short time-course microarray experiments. BMC Bioinformatics, 10:146. doi: 10.1186/1471-2105-10-146.

López-Campos, J. L., Ruiz-Ramos, M., Soriano, J. B. (2013). COPD mortality rates in Andalusia, Spain, 1975-2010: a joinpoint regression analysis. International Journal of Tuberculosis and Lung Disease, 17, 131-136.

Malvezzi, M., Bertuccio, P., Levi, F., La Vecchia, C. and Negri, E. (2012). European cancer mortality predictions for the year 2012. Annals of Oncology, 23, 1044-1052.

Menéndez J.A. and Salvador B. (1991). Anomalies of the likelihood ratio test for testing restricted hypotheses. Annals of Statistics, 19, 889-898.

Menéndez, J.A., Rueda C. and Salvador, B. (1991). Conditional test for testing a face of the tree order cone. Communications in Statistics -Simulation and Computation, 20(2\&3), 751-762.

Menéndez, J. A., Rueda, C., and Salvador, B. (1992). Dominance of likelihood ratio tests under cone constraints. Annals of Statistics, 20, 2087-2099.

Meyer, M. (2008). Inference using shape-restricted regression splines. Annals of Statistics, 2, 1013-1033.

Meyer, M. and Woodruff, M. (2000). On the degrees of freedom in shape-restricted regression. Annals of Statistics, 28, 1083-1104.

Molodecky, N. A., Soon, I.S., Rabi, D.M. et al. (2012). Increasing Incidence and Prevalence of the Inflammatory Bowel Diseases With Time, Based on Systematic Review. Gastroenterology, 142, 46-54.

Morton-Jones, T., Diggle, P., Parker, L., Dickinson, H.O. and Binks, K.(2000). Additive isotonic regression models in epidemiology. Statistics in Medicine, 9, 849-59.

Muggeo, V.M.R.(2003). Estimating regression models with unknown break-points. Statistics in Medicine, 22, 3055-3071.

Muggeo, V.M.R. (2008). segmented: an R Package to Fit Regression Models with Broken-Line Relationships. R News, 8/1, 20-25. URL http://cran.r-project.org/doc/Rnews/

O'Connell, R.G., Dockree, P.M. and Kelly, S.P. (2012). A supramodal accumulation-to-bound signal that determines perceptual decisions in humans. Nature Neuroscience, 15, 17291735 .

Pan. G. (1997). Confidence subset containing the unknown peaks of an umbrella ordering. Journal of the American Statistical Association, 92 (437), 307-314

Peddada, S. D. (1997). Confidence interval estimation of population means subject to order restrictions using resampling procedures. Statistics and Probability Letters, 31, 255-265. 
Robertson, T., Wright, F.T. and Dykstra, R.L. (1988). Order Restricted Statistical Inference. Wiley.

Rueda, C. and Lombardía, M.J. (2012). Small area semiparametric additive monotone models. Statistical Modelling, 12, 527-549.

Rueda, C. (2013). Degrees of freedom and model selection in semiparametric additive monotone regression. Journal of Multivariate Analysis, 117, 88-99.

Ruiz-Medina, M.D., Espejo, R.M., Ugarte, M.D., and Militino, A.F. (2014). Functional time series analysis of spatio-temporal epidemiological data. Stoch Environ Res Risk Assess, 28, 943-954. DOI 10.1007/s00477-013-0794-y.

Shapiro, A. (1988). Towards a unified theory of inequality constraints testing in multivariate analysis. International Statistical Review, 56, 49-62

Shi, N.Z. (1988). A test of homogeneity for umbrella alternatives and tables of the level probabilities. Commun. Statist. Theory Meth., 17, 657-670.

Shively, T.S., Walker, S.G., Damien, P. (2011). Nonparametric function estimation subject to monotonicity, convexity, and other shape constraints. Journal of Econometrics 161, 166-181.

Simard, E. P., Ward, E. M., Siegel, R. et al. (2012). Cancers with increasing incidence trends in the United States: 1999 through 200. CA: A Cancer Journal for Clinicians, 62, 118-128.

Sprent, P. (1961). Some hypotheses concerning two-phase regression lines. Biometrics, 17, 634-645.

Statistical Research and Applications Branch, National Cancer Institute (2013). Joinpoint Regression Program, Version 4.0.4 - May 2013.

Strand, M., Zhang, Y. and Swihart, B. (2010). Monotone nonparametric regression and confidence intervals. Communications in Statistics: Simulation and Computation, 39(4), 828-845.

Susko, E. (2013). Likelihood ratio tests with boundary constraints using data-dependent degrees of freedom. Biometrika, 100, 1019-1023.

Thomas, S.C. (2010). Photosynthetic capacity peaks at intermediate size in temperate deciduous trees. Tree physiology, 30, 555-573.

Turner, R. (2013). Iso: Functions to Perform Isotonic Regression. R package version 0.0-12. http://CRAN.R-project.org/package=Iso.

Turner, T.R. and Wollan, P.C. (1997). Locating a maximum usin isotonic regression. Computational Statistics and Data Analysis, 25, 305-320. 
Ugarte, M.D., Goicoa, T., Etxeberria, J., Militino, A.F. and Pollán, M.(2010). Age-specific spatio-temporal patterns of female breast cancer mortality in Spain (1975-2005). Annals of Epidemiology, 20, 906-916.

Vandenberg, L.N., Colborn, T., Hayes, T.B., Heindel, J.J., Jacobs Jr, D.R., Lee, D.H., Shioda, T., Soto, A.M., vom Saal, F.S., Welshons, W.V., Zoeller, R.T. and Myers, J.P. (2012). Hormones and endocrine-disrupting chemicals: low-dose effects and nonmonotonic dose responses. Endocrine Reviews, 33, 378-455.

Wolfe, D.A. (2006). Nonparametric distribution-free procedures for order restricted alternatives. In: Ahsanullah,M., raquad, M.Z. (eds) Recent developments in order random variables. Nova Science. New York.

Wollan, P.C. and Dykstra R.L (1986). Conditional Test with an order restriction as a null hypothesis in Advances in Order Restricted Statistical Inference. Lecture Notes in Statistcs, 37, 279-295.

Zhao, L. and Peng, L. (2002). Model selection under order restrictions. Statistics and Probability Letters, 57, 301-306. 